POS PROCEEDINGS

\title{
Detector Performance Studies at a Muon Collider
}

\section{Casarsa*}

INFN Sezione di Trieste, Trieste, Italy

\section{P. Andreetto, L. Buonincontri, C. Curatolo, A. Gianelle, D. Lucchesi and L. Sestini}

INFN Sezione di Padova and University of Padova, Padova, Italy

\section{N. Bartosik and N. Pastrone}

INFN Sezione di Torino, Torino, Italy

\section{F. Collamati}

INFN Sezione di Roma, Roma, Italy

\section{Palmer}

BNL, Upton, New York, USA

\section{Aimè, C. Riccardi and I. Vai}

INFN Sezione di Pavia and University of Pavia, Pavia, Italy

\section{P. Sala}

INFN Sezione di Milano, Milano, Italy

\begin{abstract}
A Muon Collider represents a possible option for the next generation of high-energy machines. Among the technological challenges in the realization of such a machine, the mitigation of the beam-induced background is one of the most critical issues for the experiments. At the desired instantaneous luminosity, the decay rate of the circulating muons is very high, the decay products and subsequent particles from their interactions with the machine elements can reach the detector and compromise its performance. In this contribution, the results of a first preliminary study is presented of the beam-induced background effects on the detector response in the case of muon beams collisions at a center of mass energy of $1.5 \mathrm{TeV}$ and some background mitigation strategies are illustrated.
\end{abstract}

40th International Conference on High Energy physics - ICHEP2020

July 28 - August 6, 2020

Prague, Czech Republic (virtual meeting)

\footnotetext{
${ }^{*}$ Speaker
} 


\section{Introduction}

Among the projects currently under study for the post-LHC generation of particle accelerators the muon collider represents a unique machine that has the capability to provide leptonic collisions in a multi-TeV energy range. In fact, differently from electrons, muons are not affected by significant energy losses due to synchrotron radiation when they are accelerated to very high energies and bent by the collider's dipoles. Moreover, while in the case of hadronic composite-particles collisions the interactions occur between their constituents that carry only a fraction of the total energy, in leptonic collisions all the center-of-mass energy is available for the relevant short-distance processes, opening the way to an unprecedented Physics potential. A vast Physics reach is accessible to a muon collider that ranges from high precision measurements of known standard model processes in an unexplored energy regime to the possible direct detection of new heavier states up to half the collision energy [1]. In particular, a muon collider represents a gateway to the Higgs sector, allowing precise measurements of the Higgs boson couplings to fermions and bosons and the Higgs boson self-couplings and, hence, a full determination of the Higgs potential [2].

However, such a great Physics potential is accompanied by unprecedented technological challenges on the experimental side, due to the fact that muons are unstable particles. The electrons and positrons, created in muon decays, and the photons radiated by them interact with the machine elements and produce an intense flux of $O\left(10^{10}\right)$ secondary and tertiary particles (mainly photons, neutrons, electrons and positrons, hadrons, muons) that eventually reach the detector. The amount and the characteristics of the beam-induced background (BIB) depend on the collider energy and the machine optics and lattice elements. The main features of BIB particles are relatively soft momenta (a few $\mathrm{MeV}$ for the electromagnetic component, half a $\mathrm{GeV}$ for the hadronic component, and a few tens $\mathrm{GeV}$ for muons) and asynchronous arrival times to the detector with respect to the collisions [3]. The exploitation of the full physical potential that a muon collider can offer will depend on the capacity of the experiment to mitigate and cope with the beam-induced background through cutting-edge technologies and a dedicated design of the machine-detector interface (MDI) and the detector (optimized geometry, high granularity, timing information), new sophisticated algorithms for pattern recognition and reconstruction of physical objects. In the following, the results of a preliminary study will be presented of the beam-induced background effects on the detector response in the case of muon beams collisions at $\sqrt{s}=1.5 \mathrm{TeV}$ with $2 \times 10^{12}$ muons per bunch.

\section{Detector description}

The detector model used in the studies presented here is based on CLIC's detector concept [4] with the vertex detector and the MDI adapted to cope with the harsher background environment of a muon collider. The detector comprises a full-silicon tracking system, hermetic high-granularity electromagnetic and hadronic calorimeters, and a muon spectrometer (Fig.1). The tracking detectors and the calorimeters are immersed in a 4-Tesla solenoidal magnetic field.

The tracking system consists of a vertex detector (VXD), an inner tracker (IT) and an outer tracker (OT). The VXD is instrumented with double-layer sensors arranged in four cylindrical shells at radial distances from the beamline between 3.1 and $10.2 \mathrm{~cm}$ and four endcap disks on each side at $|z|=8,12,20,28 \mathrm{~cm}$. Its $50-\mu \mathrm{m}$ thick silicon sensors are segmented in $25 \times 25 \mu \mathrm{m}^{2}$ pixels. The IT 


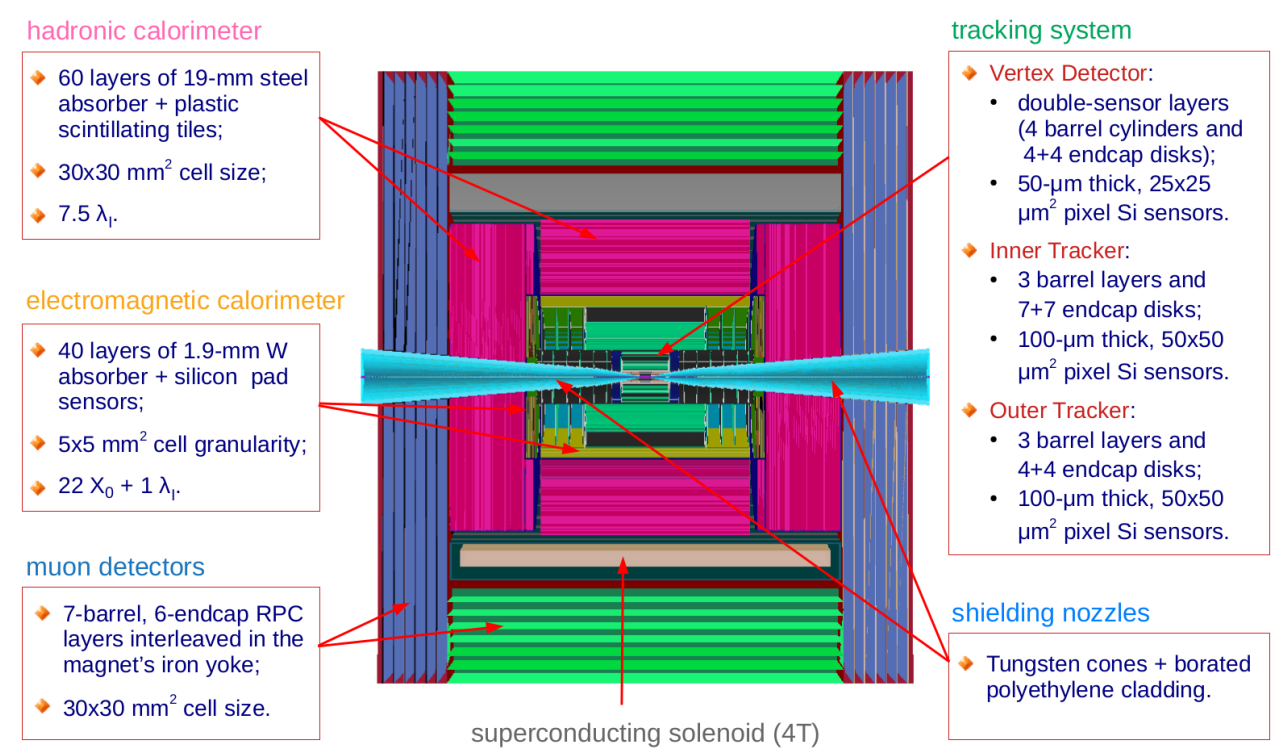

Figure 1: Cross section of the detector with a description of the subdetector components.

and OT feature $100-\mu \mathrm{m}$ thick, $50 \times 50 \mu \mathrm{m}^{2}$ pixelated silicon sensors. The IT has three barrel layers between 12.7 and $55.4 \mathrm{~cm}$ and seven disks on each side, covering the $|z|$ range between 52.4 and $219 \mathrm{~cm}$. The OT has three barrel layers placed at radii between 81.9 and $148.6 \mathrm{~cm}$ and four disks on each side between $|z|=131$ and $219 \mathrm{~cm}$. A time resolution $\sigma_{t}$ of $50 \mathrm{ps}(100 \mathrm{ps})$ is assumed for the VXD (IT and OT). The electromagnetic (ECAL) and hadronic (HCAL) calorimeters are sampling calorimeters. The ECAL consists of 40 layers of $1.9-\mathrm{mm}$ tungsten absorber and $5 \times 5-\mathrm{mm}^{2}$ silicon pad sensors for a total of $22 X_{0}+1 \lambda_{I}$. The HCAL has 60 layers of 19-mm steel absorber and $30 \times 30-\mathrm{mm}^{2}$ plastic scintillating tiles, which correspond to an interaction length of $7.5 \lambda_{I}$. The muon detectors cover the outermost region with seven barrel and six endcap layers of resistive plate chambers (RPC) with a cell size of $30 \times 30 \mathrm{~mm}^{2}$, interleaved in the magnet's iron return yoke.

Two tungsten cones ("nozzles") with a 5-cm borated polyethylene cladding, to mitigate the neutron flux, shield the beampipe on both sides of the interaction region (IR) between $|z|=6$ and $600 \mathrm{~cm}$ and provide an average background suppression by a factor of $\sim 500$. The shape of the nozzles, in particular the 10-degree opening angle, has been optimized for collisions at $\sqrt{s}=1.5$ $\mathrm{TeV}$. In correspondence with the IR is positioned a $2.2-\mathrm{cm}$ radius $400-\mu \mathrm{m}$ thick beryllium beampipe.

\section{Detector performance studies}

The following preliminary studies have been performed with detector full simulation and reconstruction tools based on CLIC's ILCSoft framework [5]. The 1.5-TeV beam-induced background was generated with the MARS15 software [6] for the U.S. Muon Accelerator Program's studies [7].

\subsection{Tracking system}

Being the closest detector to the beamline, the tracker is the most affected by the beam-induced background. Suitable background mitigation measures have to be adopted in order to preserve the detector performance. 

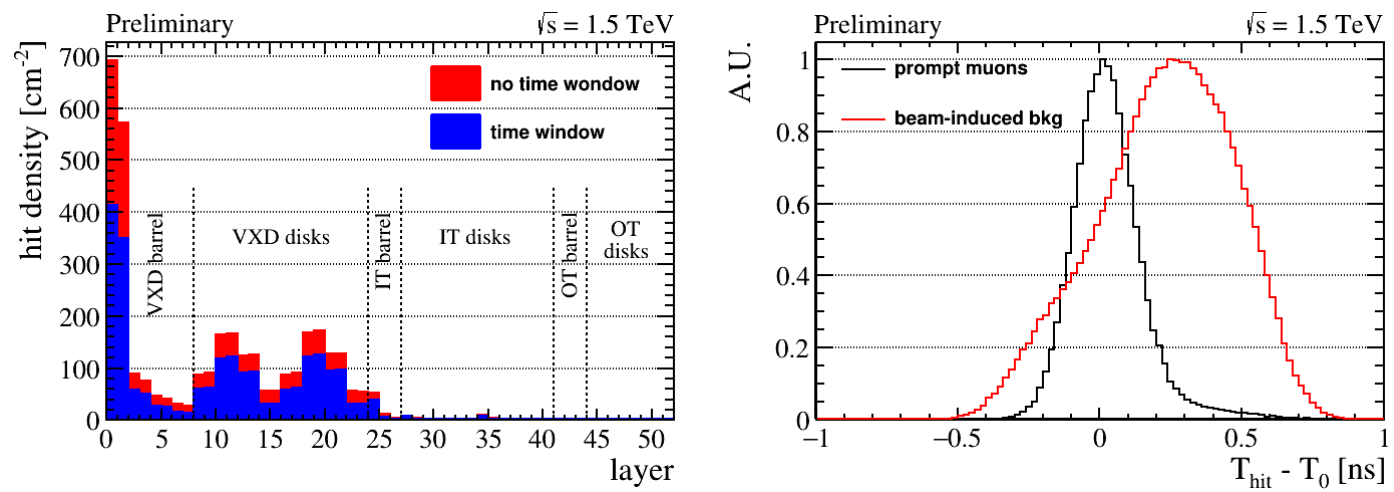

Figure 2: Left: hit density per bunch crossing on the tracker layers due to the beam-induced background. Right: hit time distributions on the first OT barrel layer for prompt muons and the BIB, where $T_{0}=\left|\vec{r}_{\text {hit }}\right| / c$.

The average hit density on the tracker layers for one bunch crossing is shown in Fig. 2 (left). A first handle to suppress the background hits is provided by the wider time spread of the hits produced by BIB particles, which are asynchronous with respect to the hard-scattering processes in the IR. An example is presented in Fig. 2 (right). A selection of the reconstructed hits that are compatible with particles originated in the IR within a $\pm 3 \sigma_{t}$ window reduces the occupancy by $\sim 30 \%$. Another powerful handle is provided by the double-layer design of the VXD Si sensors. While the products of $\mu \mu$ interactions originate in the interaction point, the BIB particles enter the detector all along the beamline and cross the VXD layers at very different angles. A correlation between the positions of hits on the two adjacent layers allows to estimate the direction of the incoming particle and reject the hits not compatible with IR particles. At the time of writing, this is still at a testing stage, but the preliminary results are very promising.

A sample of 1000 prompt muons, generated uniformly in the ranges $0.1<p_{T}<10 \mathrm{GeV}$, $0^{\circ}<\varphi<360^{\circ}, 8^{\circ}<\vartheta<172^{\circ}$, is used to assess the effect of BIB on the tracking performance. Hit pattern recognition and track finding are based on a Conformal Tracking algorithm, which is described in detail in Ref. [8]. The huge number of BIB hits, and hence the huge number of hit combinations to be fitted, makes the tracking procedure computationally very demanding. Therefore, a simplified two-step configuration of the tracking algorithm has been used with a first seeding step with VXD hits and a following seed-track extension to the IT and OT layers. The track reconstruction is performed in ten independent $\vartheta$-regions, chosen to minimize the number of hits to be processed, with boundaries at $10^{\circ}, 24^{\circ}, 33^{\circ}, 50^{\circ}, 70^{\circ}, 90^{\circ}, 110^{\circ}, 130^{\circ}, 147^{\circ}, 156^{\circ}, 170^{\circ}$. Fig. 3 shows the tracking efficiency and the $p_{T}$ resolution for prompt muons as a function of $p_{T}$ and $\vartheta$ with and without the BIB overlaid. Tracks are required to satisfy the following selection: $\chi^{2} / d o f<5, N_{\text {hits }}^{\text {trk }} \geq 6$, and $p_{T}>0.5 \mathrm{GeV}$. Track reconstruction efficiencies and $p_{T}$ resolutions in the presence of the BIB are comparable to the case with no background in the central detector region and for $p_{T}>1 \mathrm{GeV}$, whereas the performance degrades quickly at low $p_{T}$ and in the forward and backward regions, indicating that a dedicated and more sophisticated tracking strategy is needed.

\subsection{Calorimeters and muon detectors}

With the exception of two hot spots in the endcap regions closer to the beamline, the energy deposited in the calorimeters by the BIB particles at each bunch-crossing is approximately uniformly 

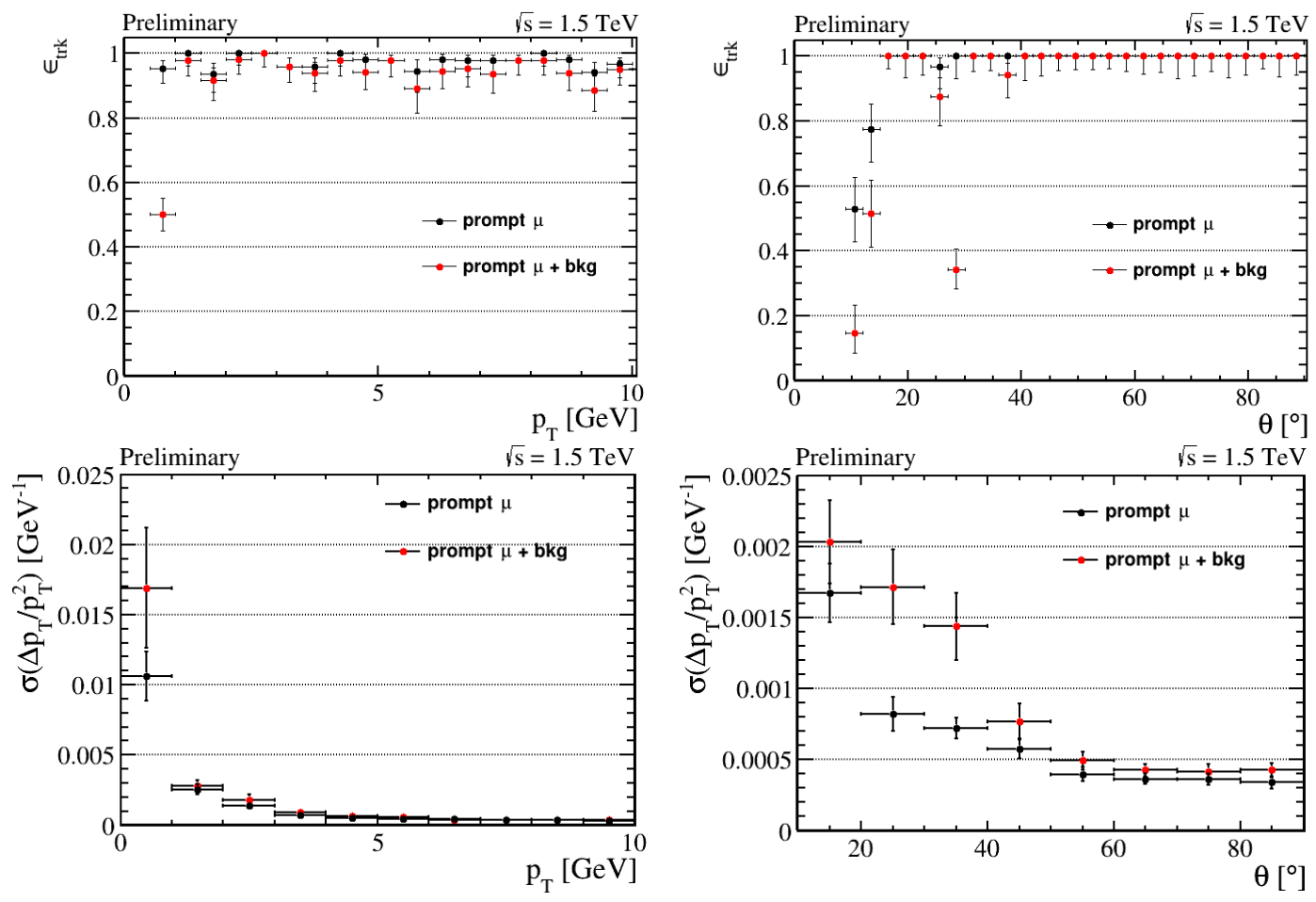

Figure 3: Tracking efficiency (top) and resolution of the reconstructed $p_{T}$ (bottom) as a function of $p_{T}$ and $\vartheta$ for prompt muons alone and prompt muons with the beam-induced background overlaid.

distributed. Under the assumption of a 5-ns readout integration window, the ECAL receives on average an energy of $\sim 2.5 \mathrm{TeV}$ mostly from photons (51\%) and electrons (48\%), whereas the HCAL collects $\sim 0.5 \mathrm{TeV}$ mainly from neutrons (99\%). Mitigation measures for the hit-clustering and jet-reconstruction algorithms are under study: timing, high granularity and fine longitudinal segmentation represent the most effective handles to mitigate the BIB effects in the calorimeters. As an example, Fig. 4 (left) compares the time distributions of ECAL barrel hits produced by a $\mu \mu$-collision process $(\mu \mu \rightarrow H v \bar{v} \rightarrow b \bar{b} v \bar{v}$ ) and the BIB. Fig. 4 (right) shows the distributions of longitudinal hit positions inside the ECAL barrel for the same two samples: particles from BIB tend to release most of their energy in the first layers of the ECAL, while the most energetic particles from collisions penetrate deeper into the calorimeter volume.

At the present time, the BIB effects on the muon detectors are being assessed. However, from the levels of hit occupancy in the HCAL regions nearby, they are expected to be minor, except for the endcap RPC's that are closer to the beamline.

\section{Conclusions}

The Muon Collider is a unique machine with an extraordinary Physics potential, that poses, however, unprecedented experimental and technological challenges on both the machine and the experiment. On the experiment side, a well-designed detector and the exploitation of state-of-the-art detector technologies and novel reconstruction and analysis techniques (like 5D tracking, advanced Particle Flow algorithms, highly-parallelized reconstruction algorithms based on GPUs like those currently 

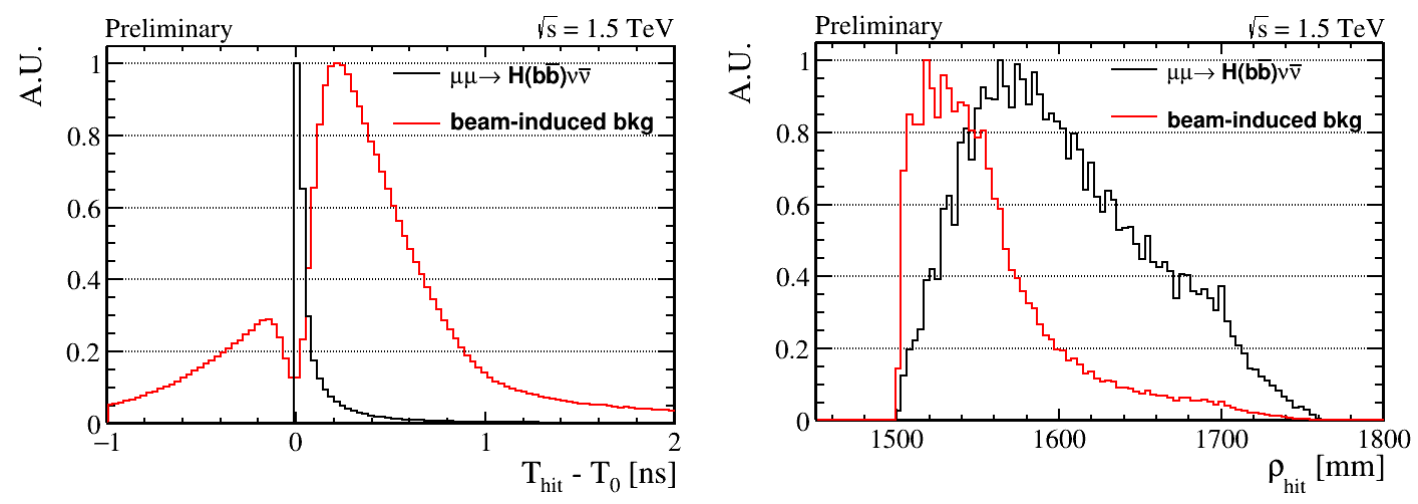

Figure 4: Time distribution (left) and longitudinal position (right) of the ECAL barrel hits for the process $\mu \mu \rightarrow H v \bar{v} \rightarrow b \bar{b} v \bar{v}$ and the BIB. $T_{0}$ is defined as $\left|\vec{r}_{h i t}\right| / c$. The normalization of distributions is arbitrary.

under development for HL-LHC) will allow to take advantage of such a potential. An example of the muon collider reach in the Higgs sector may be found in Ref. [9].

\section{References}

[1] A. Costantini et al., Vector boson fusion at multi-TeV muon colliders, arXiv:2005 . 10289v1.

[2] M. Chiesa et al., Measuring the quartic Higgs self-coupling at a multi-TeV muon collider, arXiv:2003.13628v1.

[3] N. Bartosik et al., Preliminary Report on the Study of Beam-Induced Background Effects at a Muon Collider, arXiv: 1905.03725v1.

[4] Detector Technologies for CLIC, edited by D. Dannheim, K. Krüger, A. Levy, A. Nürnberg, E. Sicking, CERN-2019-001 (CERN, Geneva, 2019) [arXiv: 1905. 02520v1].

[5] M. Frank et al., DD4hep: A Detector Description Toolkit for High Energy Physics Experiments, 2014 J. Phys.: Conf. Ser. 513 022010; F. Gaede et al., LCIO: A Persistency framework for linear collider simulation studies, CHEP-2003-TUKT001 [arXiv: physics/0306114v1]; F. Gaede, Marlin and LCCD-Software tools for the ILC, Nucl. Inst. and Methods Phys. Res. A 559 (2006) 177-180.

[6] N.V. Mokhov and C.C. James, The MARS code system user's guide version 15(2016), FermilabFN-1058-APC (2018) [DOI : 10.2172/1462233].

[7] The U.S. Muon Accelerator Program, https://map . fnal . gov.

[8] E. Brondolin et al., Conformal tracking for all-silicon trackers at future electron-positron colliders, Nucl. Inst. and Methods Phys. Res. A 956 (2020) 163304 [arXiv : 1908. 00256v1].

[9] L. Sestini et al., Higgs physics possibilities at a muon collider, Proceedings of $40^{\text {th }}$ International Conference on High Energy physics (ICHEP2020), PoS (ICHEP2020) 083. 